\title{
IMPLEMENTATION OF THE LQG CONTROLLER FOR A WIND TURBINE TOWER-NACELLE MODEL WITH AN MR TUNED VIBRATION ABSORBER
}

\author{
Maciej Rosóe \\ AGH University of Science and Technology, Department of Automatics and Biomedical Engineering, Cracow, Poland \\ e-mail:mr@agh.edu.pl \\ PaWee MarTynowicz \\ AGH University of Science and Technology, Department of Process Control, Cracow, Poland \\ e-mail: pmartyn@agh.edu.pl
}

\begin{abstract}
Vibration of a wind turbine tower is related to fatigue wear, influencing reliability of the whole structure. The current paper deals with the problem of Linear-Quadratic-Gaussian (LQG) tower vibration control using specially designed and built simulation and laboratory tower-nacelle models with a horizontally aligned, magnetorheological (MR) damper based tuned vibration absorber located at the nacelle. Force excitation applied horizontally to the tower itself, or to the nacelle, is considered. The MR damper LQG control algorithm, including the Kalman state observer and LQR (Linear-Quadratic-Regulator) controller is analysed numerically and implemented on the laboratory ground, in comparison with the system with a deactivated absorber. Simulation and experimental results are presented.
\end{abstract}

Keywords: wind turbine tower vibration, tuned vibration absorber, MR damper, LQG control

\section{Introduction}

The wind turbines sector is an emerging one nowadays. The wind load (and also sea waves load for offshore structures) that is varying in time as well as rotation of turbine elements are the major contributors to structural vibration of the tower and blades. Cyclic stress the tower is subjected to, may lead to a decrease in reliable operation time due to structure fatigue wear (Enevoldsen and Mork, 1996) or even failure accident. Tower vibration arises due to various excitation sources as variable wind/sea conditions and rotation of turbine elements (Jain, 2011). This vibration is generally lightly damped, especially considering low aeroelastic damping for the first tower lateral mode (Butt and Ishihara, 2012; Hansen et al., 2012; Matachowski and Martynowicz, 2012; Bak et al., 2012). The lateral modes are excited due to the Karman vortices, generator operation, sea waves variable load and rotating machinery unbalance rather than due to direct wind load variation/wind shear/differences in inflow conditions for each of the blades, and the blade passing effect, as for longitudinal modes. In the current research, tower vibration only is being analysed.

The solutions utilised to reduce vibration of wind turbine towers include collective pitch control, generator electromagnetic torque control, and passive/semiactive/active tuned vibration absorbers (TVAs) (Shan and Shan, 2012; Jelavić et al., 2007; Namik and Stol, 2011; Den Hartog, 1985; Oh and Ishihara, 2013; Tsouroukdissian et al., 2011; Rotea et al., 2010). TVAs are widely spread structural vibration reduction solutions for slender structures. In the standard (passive) approach, TVA consists of an additional moving mass, spring and viscous damper whose parameters are tuned to the selected (most often first) mode of the vibration (Den Hartog, 1985; Łatas and Martynowicz, 2012). Passive TVAs work well at the load conditions characterised with a single frequency to which they are tuned, but cannot adapt to a wider excitation 
spectrum (Kirkegaard et al., 2002), thus more advanced TVAs are implemented to change/tune TVA operating frequency. Among them, magnetorheological (MR) TVAs are placed (Kirkegaard et al., 2002), as the use of MR damper (instead of viscous damper) guarantees a wide range of the resistance force, fast response times, low sensitivity to temperature changes and fluid contamination, high operational robustness, and minor energy requirements (Kciuk and Martynowicz, 2011; Lord Rheonetic, 2002; Sapiński and Rosół, 2007, 2008; Snamina and Sapiński, 2011; Sapiński and Martynowicz, 2005) as compared to active systems. Simulations and experiments show that the implementation of the MR damper in the TVA system may lead to further vibration reduction in relation to the passive TVA (Martynowicz, 2014b, 2015, 2016; Koo and Ahmadian, 2007).

Within the scope of the current research, there have been specially developed and built tower-nacelle simulation and laboratory models in which all turbine components (nacelle, blades, hub, shaft, generator and possibly gearbox) are represented by nacelle concentrated mass and mass moments of inertia. Regarding variable geometric configuration of the structure resulting from changing rotor angular position, more detailed FEM analysis has been conducted using the full structure model, which demonstrated negligible influence of the rotor angle on tower structural dynamics (Matachowski and Martynowicz, 2012). Both simulation model and laboratory test rig of wind turbine tower-nacelle system give the possibility to model tower vibration under various aerodynamic, hydrodynamic, mechanical unbalance, changeable electromagnetic load, excitation sources (as mentioned before), etc. A horizontal concentrated force generated by the modal shaker may be applied either to the nacelle $P(t)$ or to the tower itself at half of its height $F(t)$. With the use of the MR damper, dedicated control solutions may be realised, in comparison to the system without TVA (i.e. TVA 'locked'). Previous research showed the effectiveness of the ground hook control and its modification, sliding mode control, linear and nonlinear damping, adaptive control and open-loop system with various MR damper constant input current values (Martynowicz, 2014b, 2015, 2016). The Linear-Quadratic-Gaussian (LQG) control approach implementation is analysed within the scope of the current paper. The first bending mode of vibration is analysed here only, as higher modes reduction capabilities with MR TVA located at the nacelle are minor (Martynowicz, 2014b, 2015).

Most of the applications of the LQG controller concern control of civil structures (buildings) excited by severe earth quakes or strong winds. The existing solutions of the LQG semiactive control algorithm use, most frequently, the mathematical model of the analysed mechanical structure (Dyke et al., 1996a,b; Asai et al., 2013; Wang and Dyke, 2013). In opposition to the Linear-Quadratic-Regulator (LQR) algorithm, they do not need measurement of the full-state for all DOFs. The Kalman state observer is responsible for the estimation of unmeasurable state variables, based on the measured positions or accelerations. In many cases, obtaining a sufficiently accurate model is difficult, therefore some authors proposed model-free LQG semiactive control algorithms which do not need an accurate mathematical model (Asai and Spencer Jr., 2014), computing LQG parameters directly from the measurement data (Hjalmarsson et al., 1998; Kawamura, 1998; Favoreel, 1999).

In the present paper, an output feedback strategy based on the measured position at a limited number of structure points is proposed. The LQG controller calculates the desired MR damper force on the basis of the state variables vector restored by the Kalman filter. The LQR problem is solved using a 'black box' input-output linear model identified on the basis of the free vibration response of the tower-nacelle experimental model. Given the measurements of the inputs and outputs of the unknown system, the matrices $\mathbf{A}, \mathbf{B}, \mathbf{C}$ and $\mathbf{D}$ of the estimate linear system are found. The model order of the 'black box' is selected considering two state variables (position and velocity) for each selected structure point.

The paper is organised as follows. In the next Section, the wind turbine tower-nacelle Comsol/Simulink model with MR TVA is introduced. Then, the laboratory test rig is presented. 
LQG controller synthesis including the Kalman filter is further described and followed by Comsol/Simulink simulation and laboratory test rig experimental results. The paper is finished with several conclusions.

\section{Wind turbine tower-nacelle model with MR TVA}

The beam modelling the tower is arranged vertically. The bottom end of the beam is fixed to the ground via additional foundation frame. A solid body modelling the nacelle is fixed to the upper end of the beam. TVA system incorporating the absorber mass, spring and MR damper is attached to the solid body representing the nacelle, and arranged horizontally. A diagram of the model, including the system of coordinates $w$ - $x$, is shown in Fig. 1a.
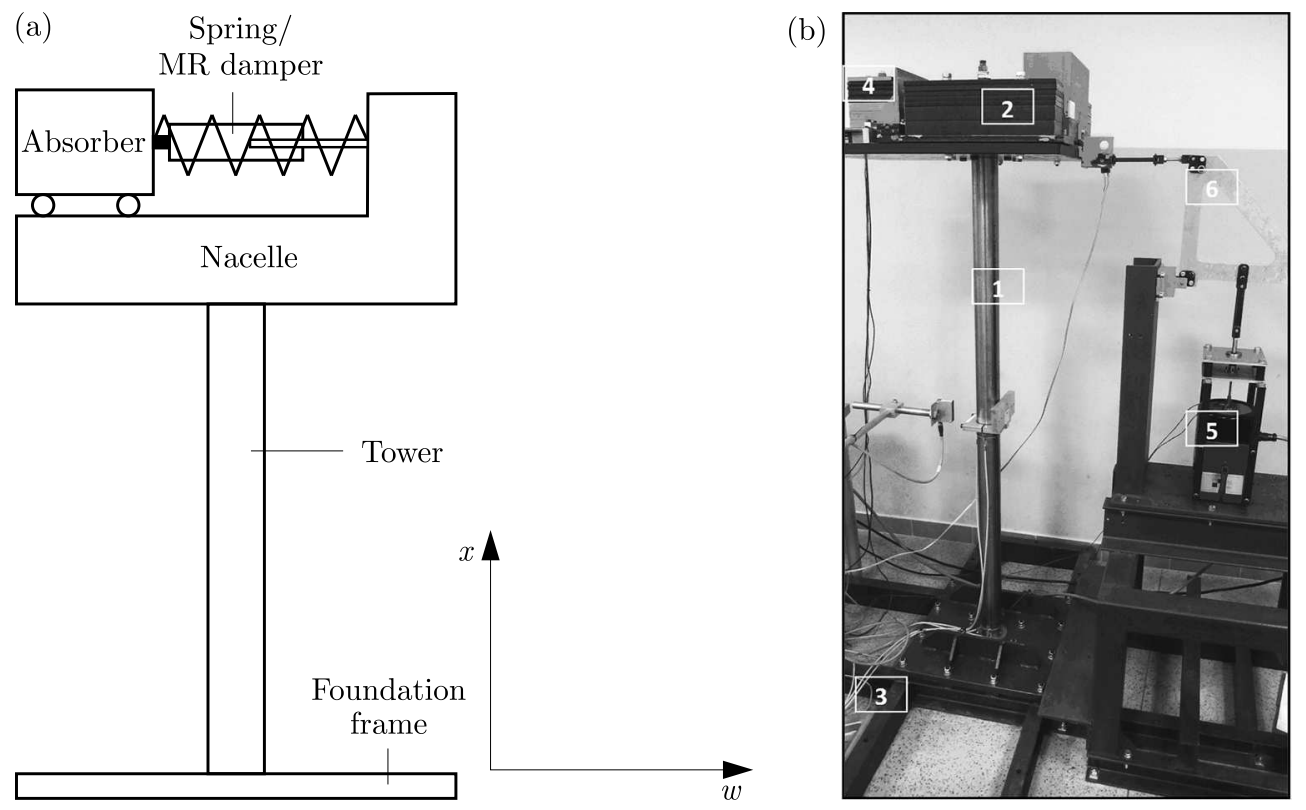

Fig. 1. (a) Diagram of the model, (b) The laboratory test rig

Based on the model assumptions and mathematical calculations results, a Comsol Multiphysics finite element method (FEM) model of the tower-nacelle system was built as a '3D Euler Beam' fixed at the bottom and free at the top, with an additional mass and mass moments of inertia defined at its top. A beam element (of length designated by $l$ ) with three nodes has been selected. The two edges are configured by applying material and cross-section parameters of the chosen tower material. The bottom node represents the tower-ground (tower-foundation) restrain, while two other nodes are 'free'. The node at the tower midpoint (at $x=x_{0}=l / 2$, see Fig. 1a), where deflection of the 2nd mode is close to maximal, is the 'load point', where the horizontal $w$-axis force $F(F(t))$ may be applied. The node at the top of the tower (at $x=x_{1}=l$ ) corresponds to the nacelle location, thus the mass and mass moments of inertia as well as concentrated load $P(P(t))$ are all assigned here.

The FEM model assumes that angles are small and cross sections are perpendicular to the bending line (Euler-Bernoulli beam model). Also, the Rayleigh model (that is precise within a narrow frequency range only) of the tower material damping is assumed. These assumptions make the developed model to be adequate for small bending angles and within the 1st bending mode frequency neighbourhood only. Also, during the model identification process (subject to separate publication), correction (lowering) of Young's modulus is necessary as the FEM EulerBernoulli beam model is stiffer than the real structure due to neglecting shear deformation and additional restrains input by the finite/limited number of elements. 
FEM Comsol Multiphysics model has been exported to MATLAB/Simulink with the 'General dynamic' option. During exporting of the 'Simulink model', forces $F$, and $P$ are specified as inputs, while the tower tip $\left(w_{x 1} / v_{x 1}\right)$ and tower midpoint $\left(w_{x 0} / v_{x 0}\right)$ displacements/velocities along the $w$-axis are defined as output signals. After the exporting, FEM tower-nacelle model is available as a MATLAB structure, and the Comsol Multiphysics model is embedded into Simulink diagram using 'COMSOL Multiphysics Subsystem' block. Thus, all 18 FEM model degrees of freedom are a part of the Simulink state vector (COMSOL, 2008).

The MR TVA model is implemented as a standard Simulink diagram. TVA model with the hyperbolic tangent model of RD-1097-1 MR damper (Martynowicz, 2015) including linear bearing guides (see Section 3) friction force, LQG controller block with MR damper optimal (demanded) force output, incorporating the Kalman filter and LQR state feedback loop, linear guides friction force compensation by an MR damper, and MR damper inverse model (to obtain demanded control current) are all embedded in the Simulink diagram. 'COMSOL Multiphysics Subsystem' block outputs are fed to the dynamics of MR TVA with mass and stiffness parameters (designated by $m$ and $k$, respectively) tuned according to Den Hartog (1985). A general structure of the regarded model is shown in Fig. 2. It contains two main blocks: the tower-nacelle system model and the MR TVA model. Its inputs are: $F, P$, while $w_{x 0}, v_{x 0}, w_{x 1}$ and $v_{x 1}$ are the outputs. The forces produced by an MR damper, spring, and bearing guides friction are all added to a force excitation $P$, (Martynowicz, 2014b, 2015).

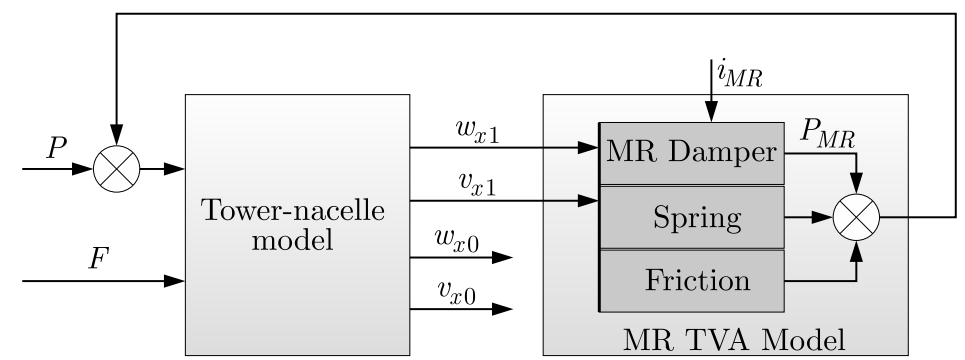

Fig. 2. Structure of the simulation model; $F, P$ - horizontal force applied at the point $x_{0}$ and point $x_{1}$, respectively, $P_{M R}-\mathrm{MR}$ damper force, $i_{M R}-$ current in the MR damper coil, $w_{x 0}, v_{x 0}$ - tower midpoint horizontal displacement and velocity, respectively, $w_{x 1}, v_{x 1}$ - tower tip (nacelle) horizontal displacement and velocity, respectively

\section{Laboratory setup}

The analysed model has to fulfil various constraints imposed by the laboratory facility and project limitations, among others adequate dimensions, strength and modal masses of the structure, and mass of the absorber corresponding to the commercially available MR damper characteristics, to enable reduction of tower deflection amplitude for the nominal MR damper stroke. It is assumed that at least partial dynamic similarity between the real-world wind turbine (Vensys 82) tower-nacelle system and its scaled model has to be fulfilled, respecting the limited laboratory space and foundation permissible load (Martynowicz, 2014a; Snamina et al., 2014; Martynowicz and Szydło, 2013). Based on all of the assumptions and analyses, a Ti. Gr. 5 alloy rod has been selected to model the wind turbine tower, while Lord's RD-1097-1 (Lord Rheonetic, 2002) MR damper has been used for TVA, and TMS 2060E lightweight electrodynamic exciter (TMS, 2010) has been selected for excitation purposes. The parameters of TVA have been tuned for the 1st bending mode of vibration (Den Hartog, 1985). After several system reconfigurations, the absorber mass has been selected to be ca. $15 \%$ of the modal mass of the 1st bending mode of the tower-nacelle model. 
The detailed analysis of a similarity relation between the laboratory model and the full-scale (Vensys 82) structure, including time and length similarity scale factors and determined geometrical and material properties of the model, was presented by Snamina et al. (2014). The conducted partial dynamic similarity analysis ensures motion similarity of a selected pair of corresponding points (tower tips).

The laboratory test rig of the wind turbine tower-nacelle with MR TVA system is presented in Fig. 1b. It is build according to the details specified above. It consists of a vertically oriented titanium alloy circular rod 1 (representing wind-turbine tower), and a system of steel plates 2 (representing the nacelle and turbine assemblies) fixed to the top of rod 1, with MR TVA embedded. The rod is rigidly mounted to steel foundation frame 3. MR TVA 4 is an additional mass moving horizontally along linear bearing guides, connected with the assembly representing the nacelle via the spring and RD-1097-1 MR damper in parallel. RD-1097-1 damper, whose force depends on the current fed to its coil, is an actuator of such a vibration reduction system. The MR TVA operates along the same direction as the vibration excitation applied to the system. The force generated by TMS 2060E exciter 5 may be applied either to rod 1 (modelling the tower) midpoint or to the system of steel plates 2 (modelling the nacelle/turbine) with the help of drive train assembly 6 of changeable leverage (enabling changeable force/displacement/velocity ranges). The excitation of the tower resulting from blade rotation, rotating machinery unbalance as well as wind thrust on the rotor may be modelled by a concentrated load $P$ applied to the nacelle/turbine, while the direct (aerodynamic, including blade passing effect, sea waves, ice, etc.) tower loads may be reduced to the resultant concentrated force $F$ applied to the tower itself (e.g. at its midpoint). All the measurements are gathered by PC with MATLAB/Simulink/RT-CON based real-time environment that is also used for the MR damper control and excitation signal generation (Martynowicz, 2015, 2016).

\section{LQG Controller synthesis}

The LQG (Linear-Quadratic-Gaussian) controllers are built for uncertain linear systems disturbed by additive white Gaussian noises, having incomplete state information (Athans, 1971). The LQG is a combination of the Kalman filter with Linear-Quadratic Regulator (LQR). The separation principle allows each part of the LQG to be designed and tested independently. The LQG controller applies to both linear time-invariant and time-varying systems. It should be noted that the LQG control problem is one of the most fundamental problems of optimal control. Application of a Kalman filter enables to restore unmeasured state variables and then use them in the LQR controller. A typical structure of the LQG regulator is shown in Fig. 3.

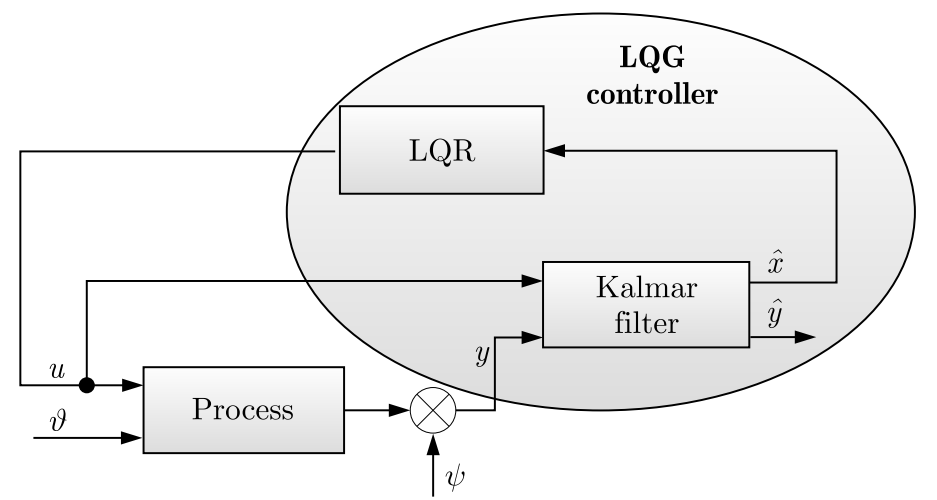

Fig. 3. Structure of a LQG controller; $\mathbf{u}$ - control input of the process, $\boldsymbol{\vartheta}$ - process noise (stochastic), $\boldsymbol{\psi}$ - measurement noise (stochastic), $\mathbf{y}$ - output of the process, $\widehat{\mathbf{y}}$ - estimation of the process output, $\widehat{\mathbf{x}}$ - estimation of the process state 
The present paper concerns a discrete-time LQG control problem. The description of the LQG controller focuses on the following discrete-time linear system of equations, modelling the tower-nacelle with MR TVA system (block 'Process' in Fig. 3)

$$
\begin{aligned}
& \mathbf{x}(k+1)=\mathbf{A x}(k)+\mathbf{B u}(k)+\boldsymbol{\vartheta}(k) \\
& \mathbf{y}(k)=\mathbf{C x}(k)+\mathbf{D u}(k)+\boldsymbol{\psi}(k)
\end{aligned}
$$

where $\mathbf{x}$ is the state vector, while the process and measurement noises, respectively: $\boldsymbol{\vartheta}(k)$ and $\boldsymbol{\psi}(k)$ are independent, zero mean, white Gaussian random processes, satisfying $\left(\mathbf{Q}_{d}, \mathbf{R}_{d}\right.$ - covariance matrices)

$$
E[\boldsymbol{\vartheta}(k)]=E[\boldsymbol{\psi}(k)]=0 \quad E\left[\boldsymbol{\vartheta}(k) \boldsymbol{\vartheta}^{\mathrm{T}}(k)\right]=\mathbf{Q}_{d} \quad E\left[\boldsymbol{\psi}(k) \boldsymbol{\psi}^{\mathrm{T}}(k)\right]=\mathbf{R}_{d}
$$

The tower-nacelle model itself ('Tower-nacelle model' block in Fig. 2), as presented in Section 2, is linearized with regard to the following four state variables: tower tip (nacelle) horizontal displacement $w_{x 1}$ and velocity $v_{x 1}$, and tower midpoint horizontal displacement $w_{x 0}$ and velocity $v_{x 0}$. All the other tower-nacelle model state variables produced by the COMSOL Multiphysics FEM model are ignored as not crucial concerning 1st (and even 2nd, regarding future applications) bending mode amplitudes detection and MR TVA control.

The LQG control algorithm can be employed for semi-active control of the tower-nacelle with MR TVA system, assuming $P_{M R}$ force as the control input. Using this algorithm, the optimal control signal $P_{M R}$, which is the force generated by an MR damper, will be obtained. The MR damper is controlled using a power interface of an analogue type. To induce the MR damper to generate the desired optimal control force, the inverse model of the MR damper is used. This model determines the relationship between the optimal value of the force $P_{M R}$, actual piston displacement (designated by $w_{x 12}$ ), actual piston velocity $\left(v_{x 12}\right)$ and MR damper current, such as: $i_{M R}=f\left(P_{M R}, w_{x 12}, v_{x 12}\right)$. However, the displacement signals of the tower-nacelle with MR TVA system are measured only - the velocity signals are not available for the LQR controller and MR damper inverse model. To solve this problem, the velocities are replaced by their estimates produced by the Kalman filter, described in detail in the next Section.

\subsection{Kalman filter}

The Kalman filter is used to restore unmeasurable variables $v_{x 1}$ and $v_{x 12}$, required to implement the LQR controller (and also the MR damper inverse model). This method provides for state (assumed in Section 4.3) of tower-nacelle with MR TVA system estimation, considering the measurement and process noises. It should be noted that in the LQG design, two Kalman filters of the same structure are used separately for $v_{x 1}$ and $v_{x 12}$.

Consider the following system

$$
\begin{aligned}
& \mathbf{z}(k+1)=\mathbf{A}_{k} \mathbf{z}(k)+\mathbf{B}_{k} \mathbf{u}(k)+\boldsymbol{\vartheta}(k) \\
& \mathbf{y}(k)=\mathbf{H}_{k} \mathbf{z}(k)+\boldsymbol{\psi}(k)
\end{aligned}
$$

where: $\mathbf{z}=\left[w_{x}, v_{x}, a_{x}\right]^{\mathrm{T}}$ is the state vector that includes displacement $w_{x}\left(w_{x 1}\right.$, or $\left.w_{x 12}\right)$, velocity $v_{x}\left(v_{x 1}\right.$, or $\left.v_{x 12}\right)$ and acceleration $a_{x}\left(a_{x 1}\right.$ or $a_{x 12}$, respectively; acceleration is estimated for future applications), while $\boldsymbol{\vartheta}(k)$ and $\boldsymbol{\psi}(k)$ are respectively the process and measurement white noises.

As only $w_{x}$ displacement is being measured, therefore the following matrices $\mathbf{A}_{k}, \mathbf{B}_{k}$, and $\mathbf{H}_{k}$ of equations (4.3) are considered (Singhal et al., 2012)

$$
\mathbf{A}_{k}=\left[\begin{array}{ccc}
1 & T_{0} & T_{0}^{2} \\
0 & 1 & T_{0} \\
0 & 0 & 1
\end{array}\right] \quad \mathbf{B}_{k}=\left[\begin{array}{l}
0 \\
0 \\
0
\end{array}\right] \quad \mathbf{H}_{k}=\left[\begin{array}{lll}
1 & 0 & 0
\end{array}\right]
$$


where $T_{0}$ is the sampling period of the LQG control algorithm (in the simulations and experiments, $T_{0}=0.001 \mathrm{~s}$ is assumed). For the calculation purposes, the following values of the covariance matrices $\mathbf{Q}_{k}, \mathbf{R}_{k}$ are assumed ( $r$ is a constant value, Singhal et al., 2012)

$$
\mathbf{Q}_{k}=\left[\begin{array}{ccc}
T_{0}^{5} / 20 & T_{0}^{4} / 8 & T_{0}^{3} / 6 \\
T_{0}^{4} / 8 & T_{0}^{3} / 3 & T_{0}^{2} / 2 \\
T_{0}^{3} / 6 & T_{0}^{2} / 2 & T_{0}
\end{array}\right] \quad \mathbf{R}_{k}=[r]
$$

The considered Kalman filter algorithm consists of two basic steps: prediction and correction.

The prediction step:

$\widehat{\mathbf{z}}_{k}^{-}=\mathbf{A}_{k} \widehat{\mathbf{z}}_{k}-$ predicted value of the state $\mathbf{z}$,

$\mathbf{P}_{k}^{-}=\mathbf{A}_{k} \mathbf{P}_{k} \mathbf{A}_{k}^{\mathrm{T}}+\mathbf{Q}_{k}$ - predicted value of the covariance.

The correction step:

$\mathbf{K}_{k}=\mathbf{P}_{k}^{-} \mathbf{H}_{k}^{\mathrm{T}}\left(\mathbf{H}_{k} \mathbf{P}_{k}^{-} \mathbf{H}_{k}^{\mathrm{T}}+\mathbf{R}_{k}\right)^{-1}$ - gain of the Kalman filter,

$\widehat{\mathbf{z}}_{k}=\widehat{\mathbf{z}}_{k}^{-}+\mathbf{K}_{k}\left[w_{x}(k)-\mathbf{H}_{k} \widehat{\mathbf{z}}_{k}^{-}\right]-$optimal, estimated value of the state $\mathbf{z}\left(w_{x}(k)\right.$ is the measured displacement value at $k T_{0}$ time step),

$\mathbf{P}_{k}=\left(\mathbf{I}-\mathbf{K}_{k} \mathbf{H}_{k}\right) \mathbf{P}_{k}^{-}$- optimal, estimated value of the covariance ( $\mathbf{I}$ is the identity matrix). The above algorithm has been implemented in form of a Simulink diagram. The Kalman filter was tested experimentally on the laboratory test rig. The tower-nacelle with MR TVA system was excited with a chirp-type force of amplitude $130 \mathrm{~N}$ applied at the point $x_{0}(A(F)=130 \mathrm{~N})$. The frequency was changing from $35 \mathrm{~Hz}$ to $1 \mathrm{~Hz}$. Figures $4 \mathrm{a}$ and $4 \mathrm{~b}$ present comparison of the displacements and velocities time responses of the tower-nacelle with MR TVA system determined from the experiment and estimated by the Kalman filter. The estimated velocity $v_{x 1}$ is compared to the one calculated by the Euler method.

Regarding the displacements, time responses practically coincide (Fig. 4a). Analysis of the velocity signals (Fig. 4b) shows the advantage of the Kalman filter over the simple-differential velocity calculation method.
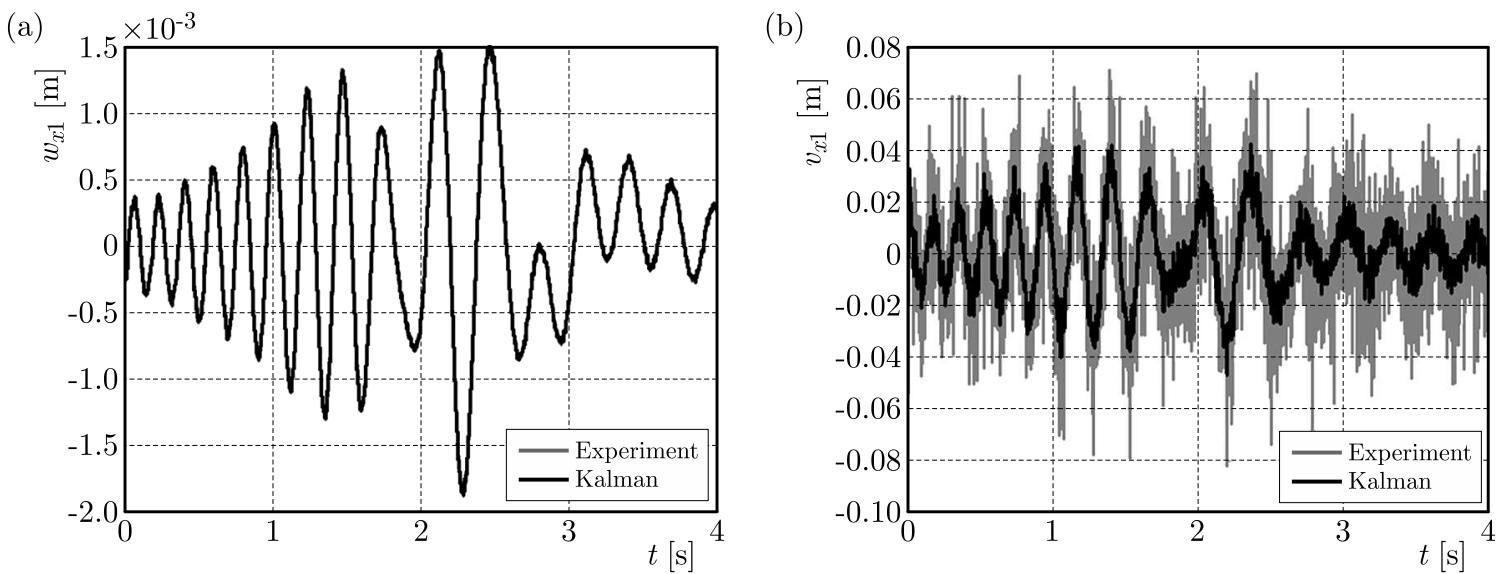

Fig. 4. Comparison of the time responses $w_{x 1}$ (a) and $v_{x 1}$ (b)

\subsection{Linearization of the wind turbine tower-nacelle model}

The purpose of the linearization procedure is to obtain a discrete linear model of towernacelle system only. The linearization procedure has been carried out using Ident tool from MATLAB Optimization toolbox. The main parameters for a black-box linear model of the Ident tool are set as follows (Ljung, 2015):

- Model structure: general linear state-space model of the 4th order. 
- Focus: simulation which approximates dynamics of the model (the transfer function from measured inputs to outputs) with a norm that is given by the input spectrum.

- Estimating method: prediction-error minimization (PEM).

To start the linearization procedure, the reference data are required. These data, describing the relationship between $w_{x 1}, v_{x 1}, w_{x 0}, v_{x 0}$ and the MR damper force $P_{M R}$, may be obtained by performing a simulation test using the nonlinear model of the tower-nacelle system with $P_{M R}$ force as the input. Such a test has been carried out for free vibration of the tower-nacelle model with non-zero initial conditions for the $w_{x 1}, v_{x 1}, w_{x 0}$ and $v_{x 0}$ signals, and for the $P_{M R}$ force changing randomly every $0.200 \mathrm{~s}$ in the range of $\pm 10 \mathrm{~N}$. An exemplary $P_{M R}$ pattern used for the linearization procedure is shown in Fig. 5. The continuous system was discretised using a zero-order hold with $T_{0}=0.001 \mathrm{~s}$.

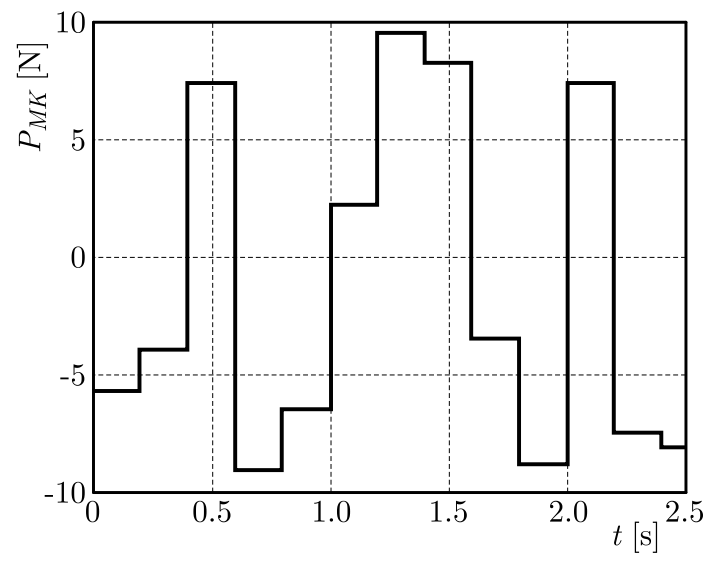

Fig. 5. An exemplary $P_{M R}$ time pattern for the linearization

Figures 6 and 7 show comparison of the reference data (nonlinear model responses, solid lines) and linear model responses of the tower midpoint and tower tip (nacelle) displacements and velocities. The numbers indicating the best fit values are given below each figure (the maximum value is 100). As can be observed, the fit level is high - it exceeds the value of 90 for each state variable. Therefore, it can be concluded that the obtained linear model can be used to implement the LQG regulator.
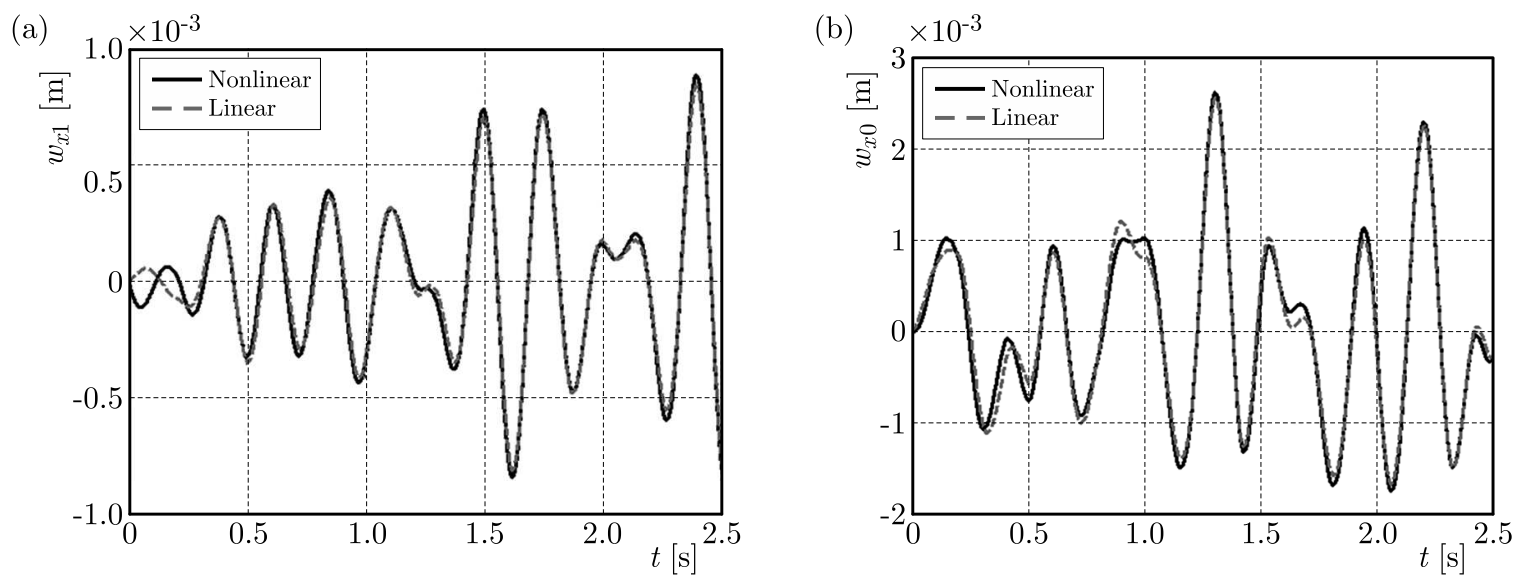

Fig. 6. Comparison of the responses $w_{x 1}$ (a) and $w_{x 0}$ (b) of nonlinear and linear models; best fits: 94.52 (a) and $95.73(\mathrm{~b})$ 

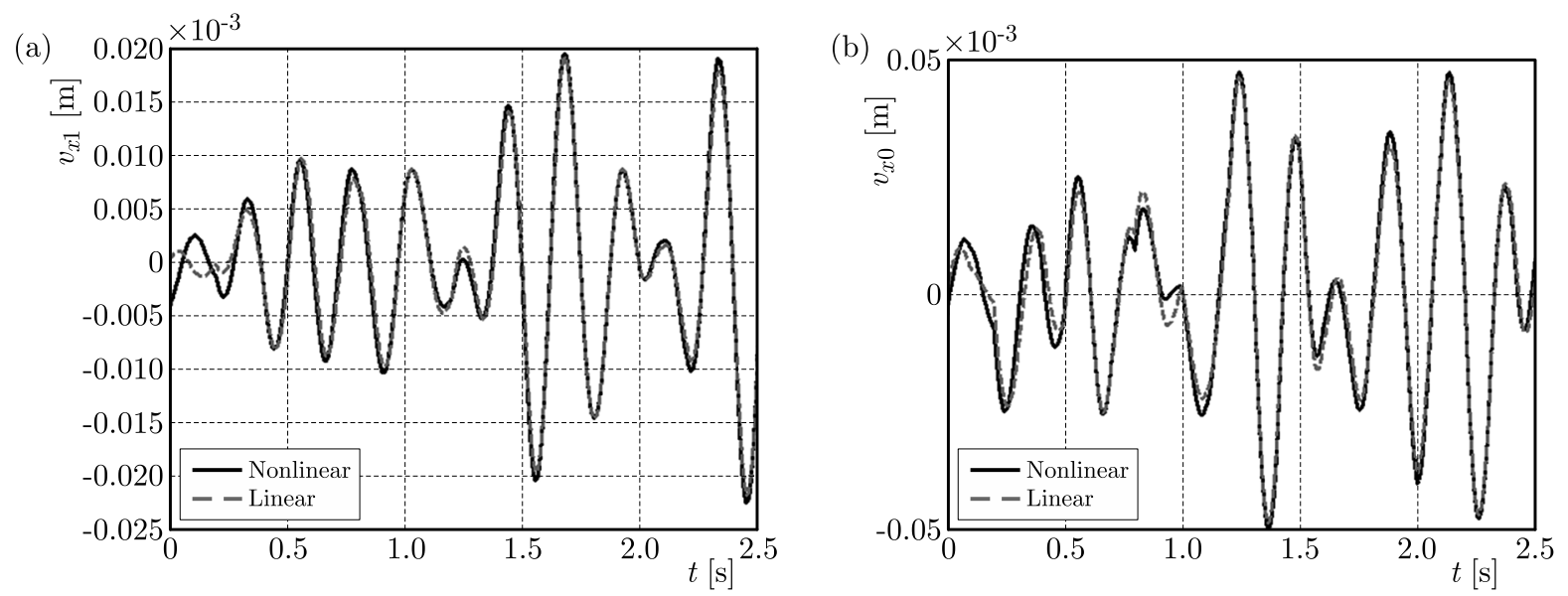

Fig. 7. Comparison of the responses $v_{x 1}$ (a) and $v_{x 0}$ of nonlinear and linear models; best fits: 94.16 (a) and 94.94 (b)

\subsection{DLQR controller}

The Discrete Linear-Quadratic Regulator (DLQR) is a state-feedback controller defined for a discrete-time state-space system. DLQR parameters are calculated by solving the optimal problem called the discrete LQR problem. This problem is defined for system dynamics described by a set of linear differential equations and a quadratic cost function.

In this paper, synthesis of a DLQR for a dynamical system described by equations (4.1) is presented. The DLQR optimization problem is solved using dlqr.m function (or dlqry.m) from MATLAB/Simulink Optimization toolbox. The dlqr. $m$ function calculates the optimal gain matrix $\mathbf{K}_{d}$ such that the state-feedback control $\mathbf{u}(k)$ (optimal MR damper force $P_{M R}$ )

$$
\mathbf{u}(k)=-\mathbf{K}_{d} \mathbf{x}(k)
$$

with the assumed state $\mathbf{x}(k)=\left[w_{x 1}(k), v_{x 1}(k), w_{x 12}(k), v_{x 12}(k)\right]^{\mathrm{T}}\left(w_{x 0}\right.$ and $v_{x 0}$ are omitted here being proportional to $w_{x 1}(k)$ and $v_{x 1}(k)$, respectively, for the 1st bending mode frequency neighbourhood regarded), minimizes the quadratic cost function

$$
J=\sum_{k=1}^{\infty}\left[\mathbf{x}^{\mathrm{T}}(k) \mathbf{Q} \mathbf{x}(k)+\mathbf{u}^{\mathrm{T}}(k) \mathbf{R u}(k)+2 \mathbf{x}^{\mathrm{T}}(k) \mathbf{N u}(k)\right]
$$

where: $\mathbf{Q}=\mathbf{Q}^{\mathrm{T}} \geqslant \mathbf{0}, \mathbf{R}=\mathbf{R}^{\mathrm{T}}>\mathbf{0}$ and $\mathbf{N}=\mathbf{N}^{\mathrm{T}} \geqslant \mathbf{0}$.

The DLQR parameters are calculated for the following forms of the matrices $\mathbf{Q}$ and $\mathbf{R}$, occurring in quality factor

$$
\mathbf{Q}=\operatorname{diag}[100,10,100,10]
$$

with different $R$ values as shown below (denoted by the LQG1), and

$$
\mathbf{Q}=\operatorname{diag}[3000,300,300,30]
$$

with $R$ equal to 0.0005 (denoted by $L Q G 2$ ). The $\mathbf{N}$ matrix is set to zero for both LQG1 and LQG2.

The element values of matrices (4.6) and (4.7) are tuned with emphasis put on the stabilization of the tower-nacelle system position and limited MR damper stroke. Hence, $Q_{11}$ and $Q_{33}$ elements of matrices (4.6), (4.7) are ten times greater than elements $Q_{22}$ and $Q_{44}$, respectively, while tower-nacelle stabilization purpose dominates over MR damper stroke limitation for LQG2 concept. The maximum control value is limited by the matrix $\mathbf{R}$. 
As the results of calculations, the following values of the DLQR gain $\mathbf{K}_{d}$ are achieved.

- LQG1 controller

$$
\mathbf{K}_{d}=\left\{\begin{array}{llll}
{\left[\begin{array}{llll}
-74.3077 & -11.0044 & -65.6904 & 275.6049
\end{array}\right]} & \text { for } \quad R=0.0001 \\
{\left[\begin{array}{llll}
-45.75 & -5.32 & -46.26 & 184.99
\end{array}\right]} & \text { for } \quad R=0.0002 \\
{\left[\begin{array}{llll}
-23.7730 & -2.2100 & -28.3578 & 104.9820
\end{array}\right]} & \text { for } \quad R=0.0005
\end{array}\right.
$$

- LQG2 controller

$$
\mathbf{K}_{d}=\left[\begin{array}{llll}
-179.81 & -59.13 & -29.48 & 289.19
\end{array}\right]
$$

In the next step, the DLQR controller and Kalman filter are integrated, forming the LQG controller. The integration stage has been executed according to the scheme shown in Fig. 8 (carets indicate estimates).

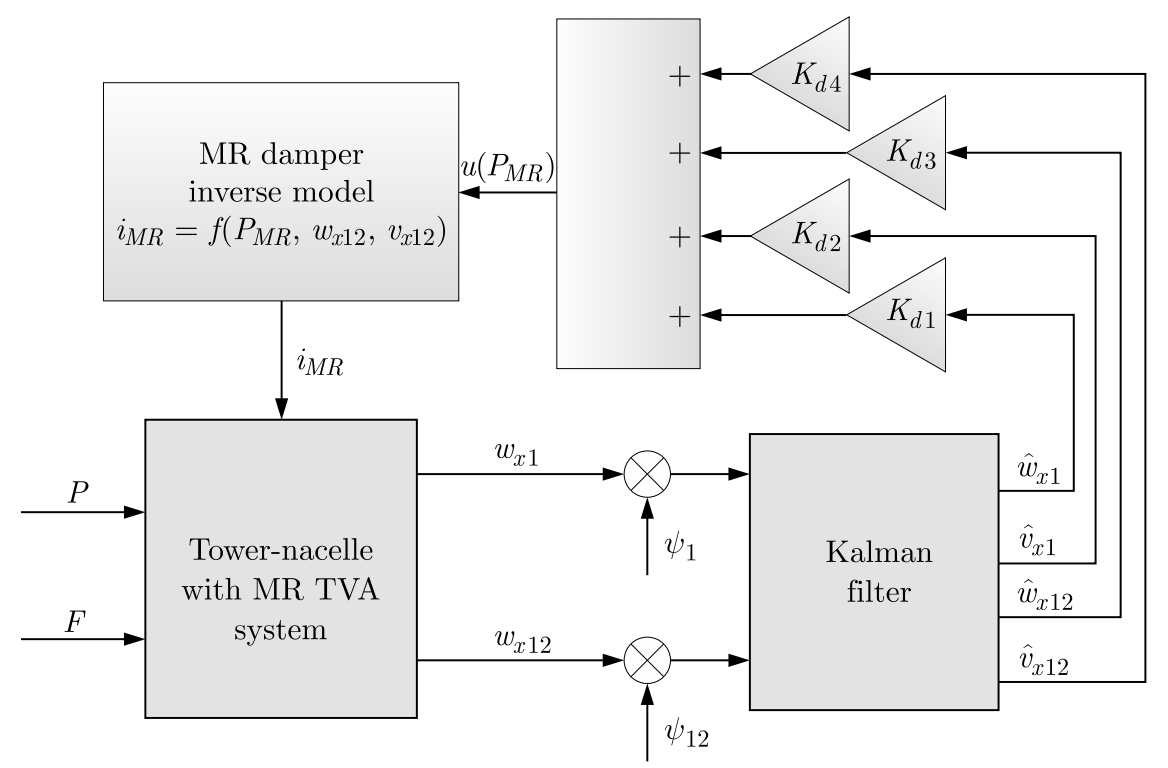

Fig. 8. Structure of the integrated LQG controller

\section{Simulation analysis}

Within the scope of the simulation analysis, time and frequency characteristics have been obtained. The latter, determined for sine excitation series applied either to the nacelle (excitation force amplitude equal to $A(P)=61 \mathrm{~N})$ or to the tower midpoint $(A(F)=150 \mathrm{~N})$ are presented below (Figures 9 and 10, see headers). Figures 9a and 10a present output frequency response functions of amplitude $A\left(w_{x 0}\right)$. Figures $9 \mathrm{~b}$ and $10 \mathrm{~b}$ show the output frequency response functions of amplitude $A\left(w_{x 1}\right)$. The information present in the legends of all the following figures has the respective meaning: 'inv' confirms incorporation of the MR damper inverse model, while 'Fr' refers to linear guides friction force (of ca. $1 \mathrm{~N}$ ) compensation by the MR damper (both of them as described in Section 2).

As can be observed, the $A\left(w_{x 1}\right)$ amplitude output frequency response functions present ca. three times greater values than the respective $A\left(w_{x 0}\right)$ functions. For LQG1 case, an increase in the control weighting value $R$ results in lower feedback gain vector $\mathbf{K}_{d}$ modulus, and so in less stiffness and less damping present between the protected structure and the absorber. This, in turn, is apparent as higher two maxima amplitudes and lower in-between the two 
maxima response. The LQG2 case, characterised with higher weights in $Q$ (especially the weights concerning the tower tip displacement $w_{x 1}$ and velocity $\left.v_{x 1}\right)$ and $R=0.0005$, produces frequency response functions similar to LQG1 controller with the lowest $R(R=0.0001)$.

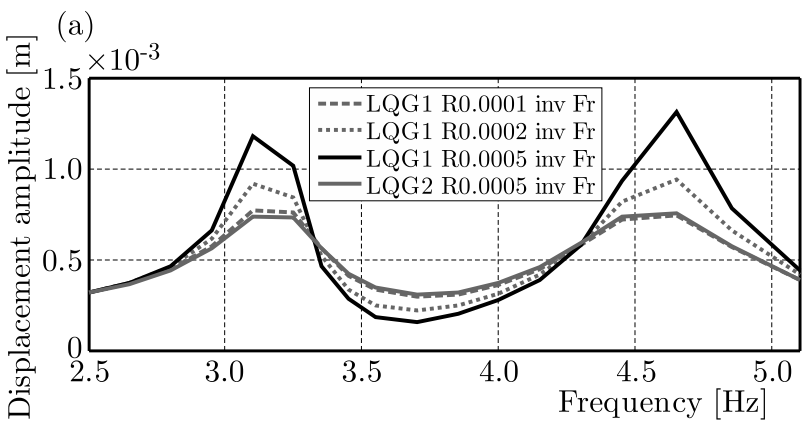

(b)

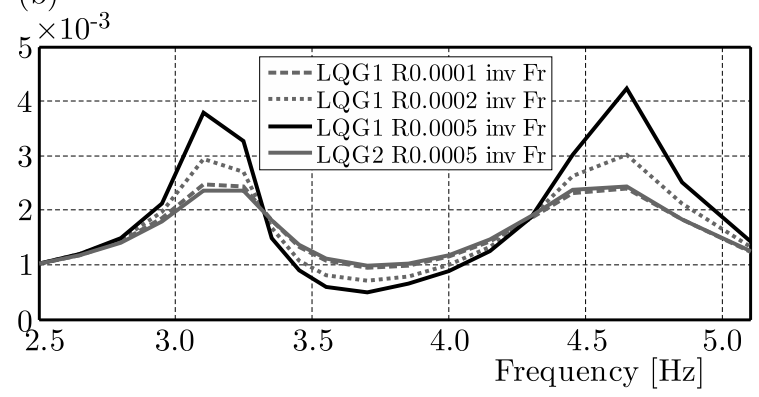

Fig. 9. (a) $A\left(w_{x 0}\right)$ and (b) $A\left(w_{x 1}\right)$ output frequency responses; $A(P)=61 \mathrm{~N}$

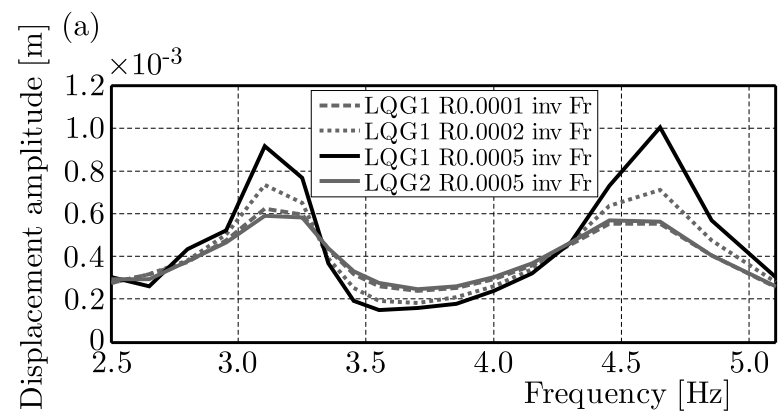

(b)

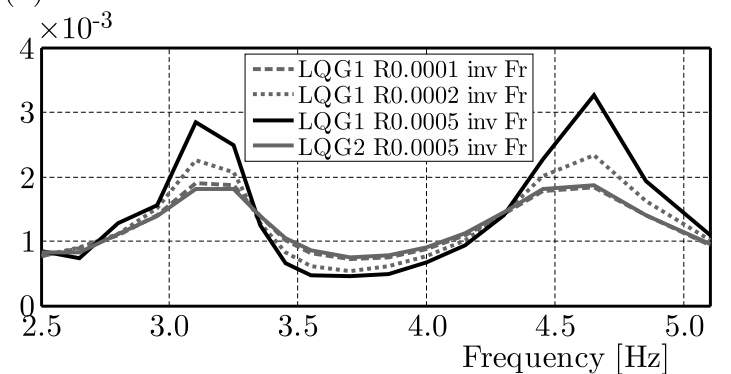

Fig. 10. (a) $A\left(w_{x 0}\right)$ and (b) $A\left(w_{x 1}\right)$ output frequency responses; $A(F)=150 \mathrm{~N}$

\section{Experimental analysis}

The experimental analysis comprised determination of time and frequency characteristics. The first was a free response test of displacement $w_{x 1}$, obtained for the MR TVA system with selected LQG controllers and the system with MR TVA 'locked' (Fig. 11). According to the

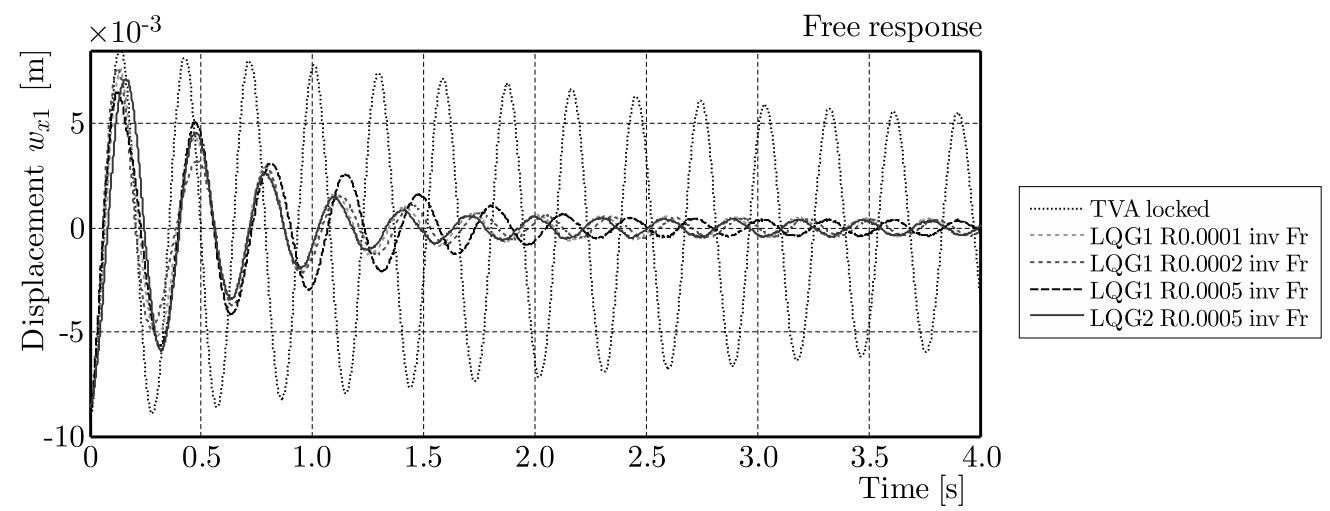

Fig. 11. Time series of displacement $w_{x 1}$ - free response of the MR TVA system with LQG controllers and the system with MR TVA 'locked'

constraints of the laboratory facility, the frequency characteristics (Figs. 12-14) were determined for sine excitation series applied either to the nacelle $(A(P)=61 \mathrm{~N}$; LQG1 system with $R=0.0001$ and $R=0.0002)$ or to the tower midpoint $(A(F)=150 \mathrm{~N}$; LQG1 and LQG2 systems with $R=0.0005)$. Figures $12 \mathrm{a}$ and $13 \mathrm{a}$ present the output frequency response functions 
of $A\left(w_{x 0}\right)$ amplitude. Figures $12 \mathrm{~b}$ and $13 \mathrm{~b}$ present the output frequency response functions of $A\left(w_{x 1}\right)$ amplitude. Figure 14 presents output frequency response functions of $A\left(w_{x 0}\right)$ and $A\left(w_{x 1}\right)$ amplitudes for the system without TVA (MR TVA 'locked').

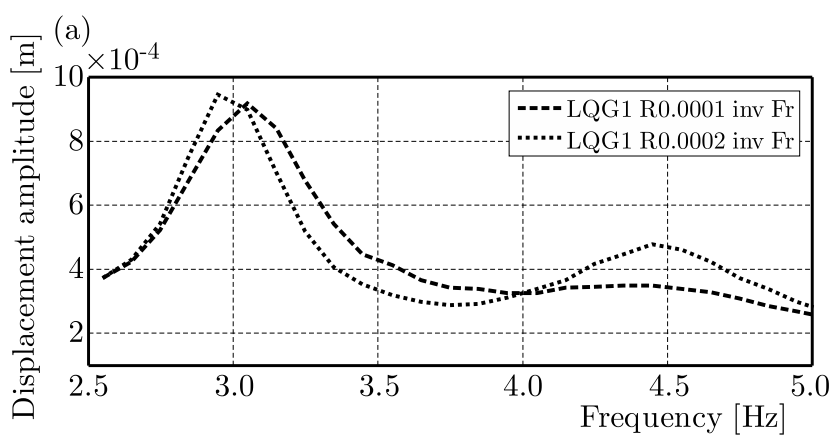

(b)

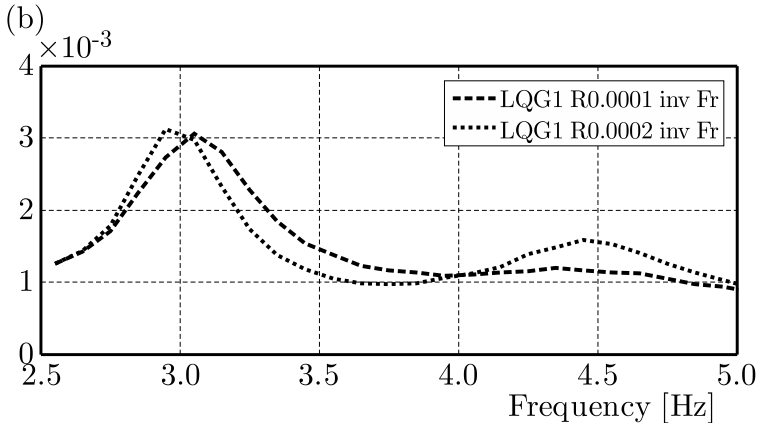

Fig. 12. (a) $A\left(w_{x 0}\right)$ and (b) $A\left(w_{x 1}\right)$ output frequency responses; $A(P)=61 \mathrm{~N}$

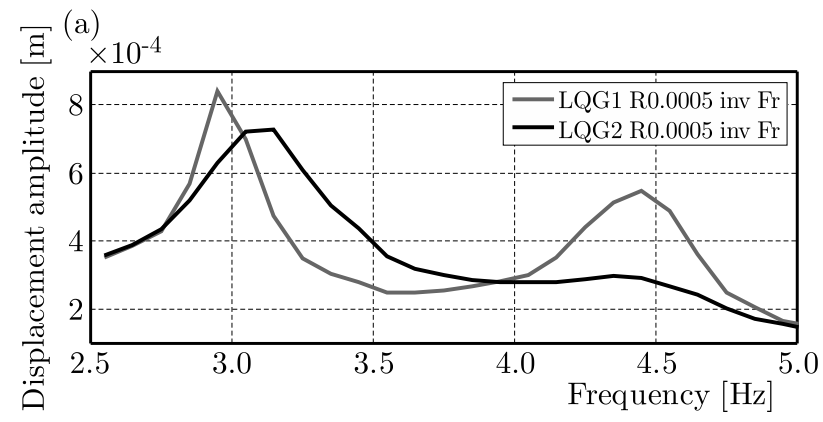

(b)

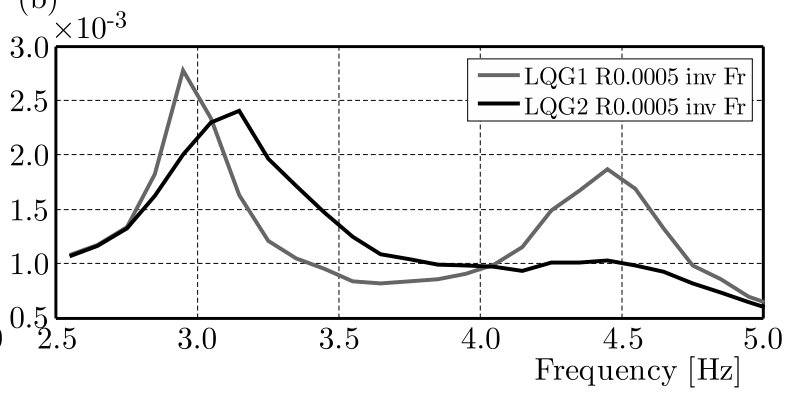

Fig. 13. (a) $A\left(w_{x 0}\right)$ and (b) $A\left(w_{x 1}\right)$ output frequency responses; $A(F)=150 \mathrm{~N}$

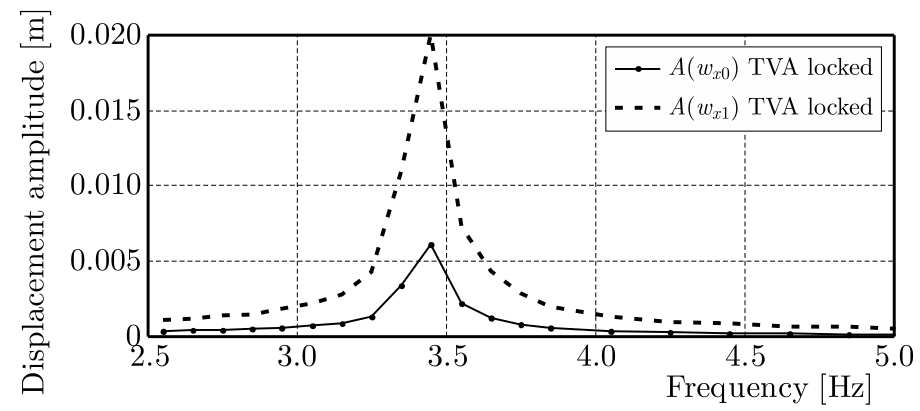

Fig. 14. $A\left(w_{x 0}\right)$ and $A\left(w_{x 1}\right)$ output frequency responses; $A(F)=150 \mathrm{~N}$

Observing the free response plots, one can conclude that LQG1 algorithm with $R=0.0001$ and LQG2 algorithm with $R=0.0005$ provide the best vibration attenuation properties. Regarding the frequency analysis, as for simulations, the $A\left(w_{x 1}\right)$ amplitude output frequency response functions exhibit ca. three times greater values than the respective $A\left(w_{x 0}\right)$ functions. LQG1 algorithm with $R=0.0001$ and $R=0.0002$ produces comparable results for the excitation series applied to the nacelle, with two maxima apparent for $R=0.0002$ case. For the excitation applied to the tower midpoint, LQG1 and LQG2 algorithms (both with $R=0.0005$ weighting value) collation may suggest the latter solution (LQG2) to be preferable, with noticeably lower two maxima amplitudes; however, in-between the two maxima range is better attenuated for LQG1. 


\section{Conclusion}

The obtained results prove the effectiveness of the LQG controller for the considered application. On the basis of the laboratory experiments, almost 10-fold reduction of the displacement $w_{x 1}$ amplitude has been observed for the LQG2 system in comparison to the system without MR TVA (TVA 'locked') - see Figs. 13b and 14. Implementation of the LQG controller combines the benefits of the LQR state feedback (including system linearization) with noise compensation by the Kalman filter.

Based on the analyses, simulations and laboratory model measurements presented in this paper, and considering force scale factor determination (Snamina and Martynowicz, 2014) in combination with the previous results (Snamina et al., 2014), direct calculation of demanded control signal for a real-world full scale vibration reduction system/MR TVA is possible as well as the calculation of the real-world wind turbine structural deflection and acceleration amplitudes, as for Vensys 82 plant regarded (Martynowicz, 2015).

\section{Acknowledgment}

This work has been supported by the Polish National Science Centre (Narodowe Centrum Nauki) under project No. 2286/B/T02/2011/40.

\section{References}

1. Asai T., Spencer Jr. B.F., 2014, Model-free algorithms for seismic response control employing controllable dampers, Proceedings of the VI World Conference on Structural Control and Monitoring, Barcelona

2. Asai T., Spencer Jr. B.F., Iemura H., Chang C.-M., 2013, Nature of seismic control force in acceleration feedback, Structural Control and Health Monitoring, 20, 789-803

3. Athans M., 1971, The role and use of the stochastic linear-quadratic-Gaussian problem in control system design, IEEE Transaction on Automatic Control, 16, 6, 529-552

4. Bak C., Bitsche R., Yde A., Kim T., Hansen M.H., Zahle F., Gaunaa M., Blasques J., Dossing M., Wedel-Heinen J-J., Behrens T., 2012, Light rotor: the 10-MW reference wind turbine, European Wind Energy Association Annual Event, 16-19.04, Copenhagen, Denmark

5. Butt U.A., Ishihara T., 2012, Seismic load evaluation of wind turbine support structures considering low structural damping and soil structure interaction, European Wind Energy Association Annual Event, 16-19.04, Copenhagen, Denmark

6. COMSOL AB, 2008, COMSOL Multiphysics MATLAB Interface Guide, COMSOL Version 3.5a, November

7. Den Hartog J.P., 1985, Mechanical Vibrations, Dover Publications, Mineola, NY

8. Dyke S.J., Spencer Jr. B.F., Sain M.K., Carlson J.D., 1996a, A new semi-active control device for seismic response reduction, Proceedings of the 11th ASCE Engineering Mechanics Specialty Conference, 886-889

9. Dyke S.J., Spencer JR. B.F., Sain M.K., Carlson J.D., 1996b, Modeling and control of magnetorheological dampers for seismic response reduction, Smart Materials and Structures, 5, 5, $565-575$

10. Enevoldsen I., Mork K.J., 1996, Effects of vibration mass damper in a wind turbine tower, Mechanics of Structures and Machines, 24, 2, 155-187

11. Favoreel W., De Moor B., Gevers M., Van Overschee P., 1999, Closed loop model-free subspace-based LQG-design, Proceedings of the 7th Mediterranean Conference on Control and Automation (MED99), Haifa, Israel, 1926-1939 
12. Hansen M.H., Fuglsang P., Thomsen K., Knudsen T., 2012, Two methods for estimating aeroelastic damping of operational wind turbine modes from experiments, European Wind Energy Association Annual Event, 16-19.04, Copenhagen, Denmark

13. Hualmarsson H., Gevers M., Gunnarsson S., Lequin O., 1998, Iterative feedback tuning: theory and applications, IEEE Control Systems Magazine, 18, 26-4.

14. JAIn P., 2011, Wind Energy Engineering, McGraw-Hill

15. Jelavić M., Perić N., Petrović I., 2007, Damping of wind turbine tower oscillations through rotor speed control, EVER Conference 200\%, March 29-April 1, Monaco

16. KaWAmura Y., 1998, Direct construction of LQ regulator based on orthogonalization of signals: Dynamical output feedback, Systems and Control Letters, 34, 1-9

17. Kciuk S., Martynowicz P., 2011, Special application magnetorheological valve numerical and experimental analysis, [In:] Diffusion and Defect Data - Solid State Data. Pt. B, Solid State Phenomena, Vol. 17r: Control Engineering in Materials Processing, 102-115

18. KirkegaARD P.H. ET AL., 2002, Semiactive vibration control of a wind turbine tower using an MR damper, Structural Dynamics EURODYN 2001, H. Grundmann (Edit.), CRC Press

19. Koo J.H., Ahmadian M., 2007, Qualitative analysis of magneto-rheological tuned vibration absorbers: experimental approach, Journal of Intelligent Material Systems and Structures, 18

20. Łatas W., Martynowicz P., 2012, Modelling of vibration of the tower-nacelle system of a wind power plant with a dynamic damper (in Polish), Modelowanie Inżynierskie, 13, 44, 187-198

21. LJung L., 2015, System Identification Toolbox ${ }^{\text {TM }}$. User's Guide, MATLAB\&Simulink R2015a, The MathWorks Inc., USA

22. Lord Rheonetic, 2002, MR Controllable Friction Damper RD-1097-01 Product Bulletin

23. Martynowicz P., 2014a, Development of laboratory model of wind turbine's tower-nacelle system with magnetorheological tuned vibration absorber, Solid State Phenomena, 208, 40-51

24. Martynowicz P., 2014b, Wind turbine's tower-nacelle model with magnetorheological tuned vibration absorber - numerical and experimental analysis, 6WCSCM: Proceedings of the 6th Edition of the World Conference of the International Association for Structural Control and Monitoirng (IACSM), 15-17.07, Barcelona, Spain

25. Martynowicz P., 2015, Vibration control of wind turbine tower-nacelle model with magnetorheological tuned vibration absorber, Journal of Vibration and Control, doi: $10.1177 / 1077546315591445$

26. MARTYNowicz P., 2016, Study of vibration control using laboratory test rig of wind turbine tower-nacelle system with MR damper based tuned vibration absorber, Bulletin of the Polish Academy of Sciences Technical Sciences, 64, 2, 347359

27. Martynowicz P., SzydŁo Z., 2013, Wind turbine’s tower-nacelle model with magnetorheological tuned vibration absorber: the laboratory test rig, Proceedings of the 14th International Carpathian Control Conference (ICCC), 26-29.05, Rytro, Poland

28. Matachowski F., Martynowicz P., 2012, Analysis of dynamics of a wind power plant by making use of Comsol Multiphysics environment (in Polish), Modelowanie Inżynierskie, 13, 44, 209-216

29. NAmik H., And Stol K., 2011, Performance analysis of individual blade pitch control of offshore wind turbines on two floating platforms, Mechatronics, 21, 691-703

30. Oh S., Ishinara T., 2013, A study on structure parameters of an offshore wind turbine by excitation test using active mass damper, EWEA Offshore, 19-21.11, Frankfurt

31. Rotea M.A., Lackner M.A., Saheba R., 2010, Active structural control of offshore wind turbines, 48th AIAA Aerospace Sciences Meeting Including the New Horizons Forum and Aerospace Exposition, 4-7.01, Orlando, Florida 
32. Sapiński B., Martynowicz P., 2005, Vibration control in a pitch-plane suspension model with MR shock absorbers, Journal of Theoretical and Applied Mechanics, 43, 3.

33. SApiński B., Rosóє M., 2007, MR damper performance for shock isolation, Journal of Theoretical and Applied Mechanics, 45, 1, 133-146

34. Sapiński B., RosóŁ M., 2008, Autonomous control system for a 3 DOF pitch-plane suspension model with MR shock absorbers, Computers and Structures, 86, 3/5, 379-385

35. Shan W., Shan M., 2012, Gain scheduling pitch control design for active tower damping and 3p harmonic reduction, European Wind Energy Association Annual Event, 16-19.04, Copenhagen, Denmark

36. Singhal T., Harit Akshat, Vishwakarma D.N., 2012, Kalman filter implementation on an accelerometer sensor data for three state estimation of a dynamic system, International Journal of Research in Engineering and Technology, 1, 6, ISSN 2277-4378

37. Snamina J., Martynowicz P., 2014,Prediction of characteristics of wind turbine's tower-nacelle system from investigation of its scaled model, 6WCSCM: Proceedings of the 6th Edition of the World Conference of the International Association for Structural Control and Monitoirng (IACSM), 15-17.07, Barcelona, Spain

38. Snamina J., Martynowicz P., Łatas W., 2014, Dynamic similarity of wind turbine's Tower-nacelle system and its scaled model, Solid State Phenomena, 208, 29-39

39. Snamina J., SAPiński B., 2011, Energy balance in self-powered MR damper-based vibration reduction system, Bulletin of the Polish Academy of Sciences Technical Sciences, 59, 1, 75-80

40. TMS, 2010, 60 Lbf Modal Shaker, The Modal Shop Inc.

41. Tsouroukdissian A., Carcangiu C.E., Pineda Amo I., Martin M., Fischer T., Kuhnle B., Scheu M., 2011, Wind turbine tower load reduction using passive and semiactive dampers, European Wind Energy Association Annual Event, Brussels

42. WANG Y., Dyke S., 2013, Modal-based LQG for smart base isolation system design in seismic response control, Structural Control and Health Monitoring, 20, 5, 753-768 